EINDHOVEN UNIVERSITY OF TECHNOLOGY

Department of Mathematics and Computing Science

Memorandum COSOR 88-25

CONVERGENCE PROPERTIES OF

INDEFINITE LINEAR QUADRATIC

PROBLEMS WITH RECEDING HORIZON

H.L. Trentelman and J.M. Soethoudt

Eindhoven University of Technology

Department of Mathematics and Computing Science

P.O. Box 513

$5600 \mathrm{MB}$ Eindhoven

The Netherlands

Eindhoven, October 1988

The Netherlands 


\title{
CONVERGENCE PROPERTIES OF INDEFINITE LINEAR QUADRATIC PROBLEMS WITH RECEDING HORIZON
}

\author{
H.L. Trentelman \& J.M. Soethoudt \\ Faculty of Mathematics \& Computer Science \\ Eindhoven University of Technology, \\ P.O. BOX 513, \\ $5600 \mathrm{MB}$ Eindhoven, \\ The Netherlands.
}

Abstract: In this paper we study the following question: given a finite dimensional linear system together with a finite horizon (possibly indefinite) quadratic cost functional, when does the corresponding optimal cost converge to the optimal cost of the corresponding infinite horizon problem, as the length of the horizon tends to infinity? For the case that the linear quadratic problems are regular we establish necessary and sufficient conditions for this convergence to hold.

Keywords: Linear quadratic optimal control, indefinite cost, large horizon, Riccati equation. 


\section{INTRODUCTION}

The finite horizon linear quadratic control problem for the linear time-invariant system

$$
\dot{x}(t)=A x(t)+B u(t), \quad x(0)=x_{0} \in \mathbb{R}^{n}
$$

is concerned with choosing a control function u such that the cost functional

$$
J_{T}\left(x_{0}, u\right):=\int_{0}^{T} \omega(x(t), u(t)) d t
$$

is minimized. Here, $\boldsymbol{\omega}$ is a real quadratic form on $\mathbb{R}^{\mathrm{n}} \mathrm{x} \mathbb{R}^{\mathrm{m}}$ given by

$$
\omega(x, u)=x^{\top} Q x+2 u^{\top} S x+u^{\top} R u
$$

In the above expressions, $A \in \mathbb{R}^{\mathrm{nxn}}, \mathrm{B} \in \mathbb{R}^{\mathrm{nxm}}, Q \in \mathbb{R}^{\mathrm{n \times n}}, S \in \mathbb{R}^{\mathrm{m \times n}}$ and $R \in \mathbb{R}^{\mathrm{m \times m}}$. It is assumed that $Q$ is symmetric and that $R$ is positive definite. Apart from this definiteness assumption on $R$, we allow $\omega$ to be indefinite.

In order for the integral in (1.2) to be well-defined, we restrict the control functions to be elements of the class $L_{2}[0, T]$ of all $\mathbb{R}^{\mathrm{m}}$-valued functions that are square integrable over [0,T]. The optimal cost for the above problem is then defined as

$$
\mathrm{V}_{\mathrm{T}}^{+}\left(\mathrm{x}_{0}\right):=\inf \left\{J_{\mathrm{T}}\left(\mathrm{x}_{0}, \mathrm{u}\right) \quad \mathrm{u} \in \mathrm{L}_{2}[0, \mathrm{~T}]\right\}
$$

In addition to the above, the infinite horizon linear quadratic problem deals with minimizing the indefinite integral

$$
J\left(x_{0}, u\right):=\lim _{T \rightarrow \infty} J_{T}\left(x_{0}, u\right)
$$

Let $L_{2,10 c}\left(\mathbb{R}^{+}\right):=\bigcap_{I \geq 0} L_{2}[0, T]$ denote the space of all $\mathbb{R}^{m}$-valued locally square integrable functions on $\mathbb{R}^{+}$. The functional $J\left(x_{0}, u\right)$ is defined for all functions $u \in I_{2,10 c}\left(\mathbb{R}^{+}\right)$for which the limit in (1.5) exists in the sense that it is finite or infinite. This class of functions is denoted by

$$
\begin{array}{r}
U\left(x_{0}\right):=\left\{u \in L_{2,10 c}\left(\mathbb{R}^{+}\right) \mid \lim _{T \rightarrow \infty} J_{T}\left(x_{0}, u\right)\right. \text { exists in } \\
\left.\mathbb{R}^{e^{\prime}}:=\mathbb{R} \cup\{-\infty,+\infty\}\right\}
\end{array}
$$


The optimal cost for the above infinite horizon linear quadratic problem is

$$
v_{f}^{+}\left(x_{o}\right):=\inf \left\{J\left(x_{o}, u\right) \mid u \in U\left(x_{o}\right)\right\}
$$

In this paper we are interested in the question whether the optimal cost for the finite horizon problem (1.4) converges to the optimal cost for the infinite horizon problem (1.7) as $T$ tends to infinity. Of course, if the quadratic form $\omega$ is positive semi-definite then it is well-known that indeed $\mathrm{V}_{\mathrm{T}}\left(\mathrm{x}_{0}\right) \rightarrow \mathrm{V}_{\mathrm{f}}^{+}\left(\mathrm{x}_{0}\right) \quad(\mathrm{T} \rightarrow \infty)$ for all $\mathrm{x}_{0}$ (provided that $(A, B)$ is stabilizable). It turns out however that if $\omega$ is indefinite then this convergence no longer holds in general. In this paper we establish necessary and sufficient conditions for convergence to hold.

Actually, we shall treat the above question of convergence in the following, more general context. Let $N \in \mathbb{R}^{n \times n}$ be a symmetric matrix, let $T>0$ and consider the finite horizon problem with cost functional

$$
J_{T, N}\left(x_{O^{\prime}} u\right):=J_{T}\left(x_{O^{\prime}} u\right)+x^{\top}(T) N x(T)
$$

The second term in the above represents a penalty on the terminal state. The matrix $\mathbf{N}$ is allowed to be indefinite. The optimal cost associated with the latter problem is given by

$$
\mathrm{V}_{\mathrm{T}, \mathrm{N}}^{+}\left(\mathrm{x}_{0}\right):=\inf \left\{\mathrm{J}_{\mathrm{T}, \mathrm{N}}\left(\mathrm{x}_{\mathrm{O}}, \mathrm{u}\right) \quad \mid \mathrm{u} \in \mathrm{L}_{2}[0, \mathrm{~T}]\right)
$$

In addition to this finite horizon problem we consider the infinite horizon problem of infimizing (1.5) under the constraint that $\mathrm{Nx}(\mathrm{t})$ converges to zero as $t$ tends to infinity. More specifically, let

$$
U_{N}\left(x_{0}\right):=\left\{u \in U\left(x_{0}\right) \mid \lim _{T \rightarrow \infty} N x(t)=0\right\}
$$

and consider the problem of infimizing (1.5) over the class $U_{N}\left(x_{0}\right)$. The optimal cost for this problem is given by

$$
\mathrm{v}_{\mathrm{N}}^{+}\left(\mathrm{x}_{0}\right):=\inf \left\{\mathrm{J}\left(\mathrm{x}_{0}, \mathrm{u}\right) \mid \mathrm{u} \in \mathrm{U}_{\mathrm{N}}\left(\mathrm{x}_{0}\right)\right\}
$$

The latter optimization problem was studied in detail in [8]. Of course, the problem (1.7) can be reobtained from this formulation as a special case by taking $N=0$ (see also [9]). 
Another special case of $(1.10)$ is obtained by taking $N=I$, the identity matrix. This special case was treated in [10].

Now, in this paper we shall ask ourselves the question: when does the optimal cost for the finite horizon problem (1.9) converge to the optimal cost for the infinite horizon problem (1.10) as $T \rightarrow \infty$ ?

We conclude this introduction by noting that the questions to be studied here have been studied before in [2] and [11]. However, in these references only the case that both $\omega \geq 0$ and $N \geq 0$ was considered, while we intend to treat the most general case that $\omega$ and $N$ are allowed to be indefinite.

\section{THE ALGEBRAIC RICCATI EQUATION}

The characterization of the optimal costs for the infinite horizon problems (1.7) and (1.10) centers around the algebraic Riccati equation (ARE):

$$
A^{\top} K+K A+Q-\left(K B+S^{\top}\right) R^{-1}\left(B^{\top} K+S\right)=0
$$

Let $\Gamma$ be the set of all real symmetric solutions of (2.1). According to [10], if $(A, B)$ is controllable and $\Gamma \neq \varnothing$ then there is exactly one $K \in \Gamma$ such that the matrix $A_{K}:=A-B R^{-1}\left(B^{\top} K+S\right)$ has all its eigenvalues in $\mathbb{C}^{-} U \mathbb{C}^{0}$ and exactly one $K \in \Gamma$ such that $A_{K}$ has all its eigenvalues in $\mathbb{C}^{+} U \mathbb{C}^{0}$. Here, we define $\mathbb{C}^{-}\left(\mathbb{C}^{0}, \mathbb{C}^{+}\right):=\{s \in \mathbb{C} \mid \operatorname{Re} s<0,(\operatorname{Re} s=0, \operatorname{Re} s>0)$. These elements of $\Gamma$ are denoted by $k^{+}$and $K^{-}$, respectively. It can be shown that if $K \in \Gamma$ then $K^{-} \leq K \leq K^{+}$. We denote $A_{K}-$ by $A^{-}$and $A_{K}+$ by $A^{+}$.

If $M \in \mathbb{R}^{n \times n}$ then $X^{-}(M)\left(X^{0}(M), X^{+}(M)\right)$ denotes the span of all generalized eigenvectors of $M$ corresponding to its eigenvalues in $\mathbb{C}^{-}\left(\mathbb{C}^{0}, \mathbb{C}^{+}\right)$.

Let $\Omega$ denote the set of all $\mathrm{A}^{-}$-invariant subspaces of $X^{+}\left(\mathrm{A}^{-}\right)$. Let $\Delta:=\mathrm{K}^{+}-$ $\mathrm{K}^{-}$( the 'gap' of the ARE). The following result states that there exists a bijection between $\Omega$ and $\Gamma$ :

Theorem 2.1 $([10],[3],[7])$. Let $(A, B)$ be controllable and assume that $\Gamma \neq \varnothing$. If $V \in \Omega$ then $\mathbb{R}^{\mathrm{n}}=V \oplus \Delta^{-1} V^{\perp}$. There exists a bijection $\gamma: \Omega \rightarrow \Gamma$ defined by

$$
\gamma(V):=\mathrm{K}^{-} \mathrm{P} v+\mathrm{K}^{+}\left(I-\mathrm{P}_{V}\right)
$$

where ${ }^{\mathrm{P}} V$ is the projector along $\Delta^{-1} V^{\perp}:=\left\{\mathrm{x} \in \mathbb{R}^{\mathrm{n}} \mid \Delta \mathrm{x} \in \mathcal{V}^{\perp}\right\}$. If $\mathrm{K}=\gamma(V)$ then $X^{+}\left(\mathrm{A}_{\mathrm{K}}\right)=V, X^{0}\left(\mathrm{~A}_{\mathrm{K}}\right)=\operatorname{ker} \Delta$ and $X^{-}\left(\mathrm{A}_{\mathrm{K}}\right)=X^{-}\left(\mathrm{A}^{+}\right) \cap \Delta^{-1} V^{\perp}$.

If $K=\gamma(V)$ then $\mathrm{K}$ is said to be supported by $V$. 


\section{THE INEINITE HORIZON PROBLEM WITH ASYMPTOTIC CONSTRAINTS}

In this section we briefly recall the results from [8] on the infimization problem (1.10). Let $\mathscr{L}$ be a subspace of $\mathbb{R}^{\mathrm{n}}$. A symmetric matrix $K \in \mathbb{R}^{\mathrm{n} \times n}$ is called negative semi-definite on $\mathscr{L}$ if the following two conditions hold: (i) $\forall$ $\mathrm{x} \in \mathscr{L}: \mathrm{x}^{\top} \mathrm{K} \mathrm{x} \leq 0$ (ii) $\forall \mathrm{x} \in \mathscr{L}: \mathrm{x}^{\top} \mathrm{K} \mathrm{x}=0 \Leftrightarrow \mathrm{Kx}=0$.

If $V \subseteq \mathbb{R}^{n}$ and $M \in \mathbb{R}^{n \times n}$ then $\langle V \mid M\rangle$ will denote the largest M-invariant subspace of $V$. A key role in the characterization of the optimal cost $V_{N}^{+}\left(x_{0}\right)$ is played by the subspace

$$
V_{\mathrm{N}}:=\left\langle\operatorname{ker} \mathrm{N} \cap \operatorname{ker} \mathrm{K}^{-}\right| \mathrm{A}^{-}>\cap X^{+}\left(\mathrm{A}^{-}\right)
$$

observe that $V_{\mathrm{N}} \in \Omega$. Thus, with $V_{\mathrm{N}}$ there corresponds exactly one solution of the ARE. This solution $\gamma\left(V_{N}\right)$ is denoted by $k_{N}^{+}$. The following theorem is the main result of $[8]$ :

Theorem 3.1 Let $(A, B)$ be controllable. Assume that $\Gamma \neq \varnothing$ and that $K^{-}$is negative semi-definite on ker $N$. Then we have

(i) $v_{N}^{+}\left(x_{0}\right)=x_{0}^{\top} K_{N}^{+} x_{0}$ for all $x_{0} \in \mathbb{R}^{n}$.

(ii) For all $x_{0} \in \mathbb{R}^{n}$ there exists an optimal $u^{*}$ if and only if ker $\Delta \subseteq \operatorname{ker} \mathrm{N} \cap \operatorname{ker} \mathrm{K}^{-}$.

(iii) If ker $\Delta \subseteq$ ker $N \cap$ ker $\mathrm{K}^{-}$then there exists exactly one optimal input and, moreover, this input is given by the feedback control law $u=$ $-R^{-1}\left(B^{\top} K_{N}^{+}+S\right) x$.

\section{THE FINITE HORIZON PROBLEM WITH ENDPOINT PENALTY}

In this section we consider the finite horizon problem of infimizing the cost functional (1.8). We note that for the case that both $\omega \geq 0$ and $N \geq 0$ this problem is quite standard and is treated, for example, in [6]. The general case however is slightly more complicated. The following result can be found in $[1, \mathrm{p} .131]$ : 
Lemma 4.1 Suppose there exists on the interval $[0, \infty)$ a solution $K(t)=K_{N}(t)$ of the Riccati differential equation (RDE):

$$
\begin{aligned}
& \dot{K}(t)=A^{\top} K(t)+K(t) A+Q-\left(K(t) B+S^{\top}\right) R^{-1}\left(B^{\top} K(t)+S\right), \\
& K(0)=N .
\end{aligned}
$$

Then for all $T>0$ and for all $x_{0} \in \mathbb{R}^{n}$ we have

$$
V_{T, N}^{+}\left(x_{0}\right)=x_{0}^{T} K_{N}^{+}(T) x_{0}
$$

For all $T>0$ and $x_{0} \in \mathbb{R}^{n}$ there is exactly one $u^{*} \in L[0, T]$ such that $J_{T, N_{T}}\left(x_{0}, u^{*}\right)$ $=V_{T, N}^{+}\left(x_{0}\right)$. This input is given by the feedback control law $u=-R^{-1}\left(B^{\top} K(T-t)\right.$ $+S) x ; t \in[0, T]$.

It is well-known that if $\omega \geq 0$ and $N \geq 0$ then (4.1) indeed has a unique solution on $[0, \infty)$. In the general case, the RDE need not have a solution on a given interval. We do have the following:

Lemma 4.2 Suppose that $(A, B)$ is controllable and that $\Gamma \neq \varnothing$. If $N-K^{-} \geq 0$ then (4.1) has a unique solution $K_{N}(t)$ on $[0, \infty)$. In fact, $K_{N}(t)=K^{-}+D(t)$, where $D(t)$ is the unique solution on $[0, \infty)$ of

$$
\begin{aligned}
& \dot{D}(t)=A^{-\top} D(t)+D(t) A^{-}-D(t) B R^{-1} B^{\top} D(t), \\
& D(0)=N-K^{-} .
\end{aligned}
$$

(with $A^{-}=A_{K^{-}}$, see section 2).

Proof The fact that (4.2) has a unique solution $D(t)$ on $[0, \infty)$ is standard (see for example $[4$, cor. 2.4 .4$])$. It is then a matter of straightforward calculation to show that $K^{-}+D(t)$ satisfies $(4.1)$. Uniqueness of $K^{-}+D(t)$ then follows from the uniqueness of $D(t)$. D

\section{CONVERGENCE OF THE OPTIMAI COST}

In this section we shall give a formulation of our main result. Before doing this, we state the following lemma: 
Lemma 5.1 Let $\mathscr{L}$ be a subspace of $\mathbb{R}^{n}$ and let $K \in \mathbb{R}^{\text {nxn }}$ be a symmetric matrix. Then $K$ is negative semi-definite on $\mathscr{L}$ if and only if there exists a symetric matrix $N$ such that $\operatorname{ker} N=\mathscr{L}$ and $N-K \geq 0$.

Proof A proof of this can be given similar to the proof of $[8,1$ emma 3.3]. 0

Consider the problems (1.8) and (1.10). In the remainder of this section we assume that $(A, B)$ is controllable and that $\Gamma \neq \varnothing$. According to the previous lemma, if $\mathrm{N}-\mathrm{K}^{-} \geq 0$ then $\mathrm{K}^{-}$is negative semi-definite on ker $\mathrm{N}$. Conversely, if $\mathrm{K}^{-}$is negative semi-definite on ker $\mathrm{N}$ then one can always find a symmetric matrix $\mathrm{N}_{1}$ such that ker $\mathrm{N}_{1}=\operatorname{ker} \mathrm{N}$ and $\mathrm{N}_{1}-\mathrm{K}^{-} \geq 0$.

We now formulate our main result:

Theorem 5.2 Assume that $(A, B)$ is controllable, $\Gamma \neq \varnothing, N-K^{-} \geq 0$ and ker $\Delta \subseteq$ ker $N \cap$ ker $K^{-}$. Then $V_{T, N}^{+}\left(x_{0}\right) \rightarrow V_{N}^{+}\left(x_{0}\right)(T \rightarrow \infty)$ for all $x_{0} \in \mathbb{R}^{n}$ if and only if ker $\mathrm{N} \cap \operatorname{ker} \mathrm{K}^{-}$is $\mathrm{A}^{-}$-invariant.

A proof of this theorem is given in section 6 . In the remainder of this section we shall discuss some important special cases of this theorem. First we consider the case that $\mathrm{N}=0$, that is, the question whether $\mathrm{V}_{\mathrm{T}}^{+}\left(\mathrm{x}_{0}\right) \mathrm{given}$ by (1.4) converges to the optimal cost $v_{f}^{+}\left(x_{0}\right)$ given by $(1.7)$.

Corollary 5.3 Assume that $(A, B)$ is controllable, $\Gamma \neq \varnothing, \mathrm{K}^{-} \leq 0$ and ker $\Delta \leq$ ker $\mathrm{K}^{-}$. Then $\mathrm{V}_{\mathrm{T}}^{+}\left(\mathrm{x}_{0}\right) \rightarrow \mathrm{V}_{f}^{+}\left(\mathrm{x}_{0}\right)(\mathrm{T} \rightarrow \infty)$ for all $\mathrm{x}_{0}$ if and only if $k e r K^{-}$is $A^{-}$-invariant. $\square$

It is well-known that if, in addition, $\omega \geq 0$ then if $(A, B)$ is controllable we always have convergence of $\mathrm{v}_{\mathrm{T}}^{+}\left(\mathrm{x}_{0}\right)$ to $\mathrm{v}_{f}^{+}\left(\mathrm{x}_{0}\right)$. This result can be reobtained using the previous corollary in combination with the following lemma:

Lemma 5.4 Assume that $\omega \geq 0$. If $(A, B)$ is controllable then $\Gamma \neq \sigma, K^{-} \leq 0$, ker $\Delta \subseteq$ ker $\mathrm{K}^{-}$and ker $\mathrm{K}^{-}$is $\mathrm{A}^{-}$-invariant.

Proof A proof of these claims can be found in [9].

Next, we consider the special case that $N \geq 0$ and $\omega \geq 0$. It turns out that if $\omega \geq 0$ then the subspace ker $\mathrm{K}^{-}$can be calculated in terms of the matrices $A, B, Q, S$ and $R$. In fact, it can be shown that if $(A, B)$ is controllable then 


$$
\begin{aligned}
& \operatorname{ker} \mathrm{K}^{-}=\left\langle\operatorname{ker}\left(Q-S^{\top} R^{-1} S\right)\right| A-\mathrm{BR}^{-1} S> \\
& \cap\left\{X^{+}\left(A-B R^{-1} S\right) \oplus X^{0}\left(A-B R^{-1} S\right)\right\},
\end{aligned}
$$

(see [9]). The latter subspace is exactly the undetectable subspace of the pair $\left(Q-S^{\top} R^{-1} S, A-B R^{-1} S\right.$ ) (with respect to the stability set $\mathbb{C}^{-}$). Denote this subspace by $N D\left(\left(Q-S^{\top} R^{-1} S, A-B R^{-1} S\right)\right.$.

Corollary 5.5 Assume $\omega \geq 0$ and $N \geq 0$. If $(A, B)$ is controllable and ker $\Delta \subseteq$ ker $N \cap N D\left(1 Q-S^{\top} R^{-1} S, A-B R^{-1} S\right)$. Then $V_{T, N_{1}^{+}}^{+}\left(x_{0}\right) \rightarrow V_{f}^{+}\left(x_{0}\right)(T \rightarrow \infty)$ for all $x_{0}$ if and only if ker $N \cap N D\left(\left(Q-S^{\top} R^{-1} S, A-B R^{-1} S\right)\right.$ is $\left(A-B R^{-1} S\right)$-invariant. $\square$

A similar result was obtained in [2], with ' $(A, B)$ controllable' replaced by ' $(A, B)$ stabilizable' and 'ker $\Delta \subseteq \operatorname{ker} N \cap N D\left(Q-S^{\top} R^{-1} S, A-B R^{-1} S\right)$ ' replaced by ' $\Delta>0^{\prime}$. The methods used in [11] ( and [2]) to prove this result will however turn out to be completely different from ours.

Note that the results presented up to now all require $N-K^{-} \geq 0$. We now ask ourselves the question: what can we do if this is not the case? Again consider the problems (1.9) and (1.10) and assume that $K^{-}$is negative semi-definite on ker N. (see th.3.1). Now, the latter does not imply that $N$ $K^{-} \geq 0$ but according to lemma 5.1 we can find a new weighting matrix $\mathrm{N}_{1}$ such that ker $N=\operatorname{ker} N_{1}$ and $N_{1}-K^{-} \geq 0$. Clearly, $U_{N}\left(x_{0}\right)=U_{N_{1}}\left(x_{0}\right)$ for all $x_{0}$ and hence $\mathrm{v}_{\mathrm{N}_{1}}^{+}\left(\mathrm{x}_{0}\right)=\mathrm{v}_{\mathrm{N}}^{+}\left(\mathrm{x}_{0}\right)$ for all $\mathrm{x}_{0}$. We can then apply theorem 5.2 with $\mathrm{N}$ replaced by $\mathrm{N}_{1}$ and study the question whether $\mathrm{V}_{\mathrm{T}_{1} \mathrm{~N}_{1}}^{+}\left(\mathrm{x}_{0}\right)$ converges to $\mathrm{V}_{\mathrm{N}_{1}}^{+}\left(\mathrm{x}_{0}\right)=$ $\mathrm{v}_{\mathrm{N}}^{+}\left(\mathrm{x}_{\mathrm{O}}\right)$. An example of this is following: suppose we consider the problem with $N=I$. There always exist a real number $k \neq 0$ such that $k I-K^{-} \geq 0$. Of course, $V_{I}^{+}\left(x_{0}\right)=v_{k I}^{+}\left(x_{0}\right)$ for all $x_{0}$. We can then apply theorem 5.2 to obtain:

Corollary 5.6 Assume that $(A, B)$ is controllable, $\Gamma \neq \varnothing$ and $\Delta>0$. Then there exists $k \neq 0$ such that $V_{T, k I}^{+}\left(x_{0}\right) \rightarrow V_{I}^{+}\left(x_{0}\right)(T \rightarrow \infty)$ for all $x_{0}$. In fact, convergence holds for all $k \neq 0$ such that $k I \geq K^{-}$.

\section{PROOF OF THE MAIN RESULT}

Our proof of theorem 5.2 runs along a series of lemmas that we consider to be interesting in their own right. Due to lack of space the proofs of these lemmas are deferred to a future paper. Our first lemma deals with an arbitrary system $(A, B)$ and an arbitrary matrix $R>0$, independent of the previous context. Consider the standard Riccati differential equation 


$$
\begin{aligned}
& \dot{P}(t)=A^{\top} P(t)+P(t) A-P(t) B R^{-1} B^{\top} P(t), \\
& P(0)=P_{O^{\prime}}
\end{aligned}
$$

together with the standard algebraic Riccati equation

$$
\text { (6.2) } \quad A^{\top} P+P A-P B R^{-1} B^{\top} P=0 \text {. }
$$

Recall that if $P_{0} \geq 0$ and $(A, B)$ is controllable, then $(6.1)$ has a unique solution $P(t) \geq 0$ on $[0, \infty)$ (see [4]). Also, (6.2) has at least one solution $([5])$. Let $P^{+}$be the largest real symetric solution of $(6.2)$.

Lemma 6.1 Assume that $(A, B)$ is controllable and $\sigma(A) \subseteq \mathbb{C}^{+}$. Then $P^{+}>0$. For any $P_{0}>0$ we have $\lim _{t \rightarrow \infty} P(t)=P^{+}$. $\square$

Our following result again deals with the Riccati differential equation (6.1). The result is, in a sense, the converse of the previous lemma:

Lemma 6.2 Assume $P_{0} \geq 0$ and assume that $\lim _{t \rightarrow \infty} P(t)=: P \in \mathbb{R}^{n \times n}$ exists. If $P>0$ then $\mathrm{P}_{0}>0$. $\mathrm{D}$

We now return to the original context of this paper. Consider the system (1.1), together with the quadratic form $\omega$ given by (1.3). Recall that we denote $A_{K^{-}}$by $A^{-}$(see section 2 ). An important role is played by the following algebraic Riccati equation in the unknown $D$ :

$$
\text { (6.3) } \quad A^{-T} D+D A^{-}-D B R^{-1} B^{\top} D=0
$$

We make the following observation:

Lemma 6.3 Assume $(A, B)$ is controllable and $\Gamma \neq \varnothing$. Let $K \in \mathbb{R}^{n \times n}$ be symmetric. Then $\mathrm{K}$ is a solution of (2.1) if and only if $\mathrm{D}=\mathrm{K}-\mathrm{K}^{-}$is a solution of (6.3). In particular $D=0$ and $D=\Delta\left(=K^{+}-K^{-}\right)$are solutions of (6.3). In fact, 0 and $\Delta$ are the extremal solutions of $(6.3)$ in the sense that any solution of (6.3) satisfies $0 \leq D \leq \Delta$. 口

Finally, we shall need the following result:

Lemma 6.4 Assume that $(A, B)$ is controllable and $\Gamma \neq 0$. Assume that $N-K^{-} \geq 0$. Let $D(t)$ be the solution of (4.3). Then for all $t \geq 0$ we have: 
We have now collected the most important ingredients that will be used in our proof of theorem 5.2. In order to give this proof we shall make a suitable decomposition of the state space. Let $V_{N}$ be the subspace defined in ( 3.1 ). Define

$$
\begin{aligned}
& x_{1}:=v_{\mathrm{N}} \\
& x_{2}:=\operatorname{ker} \Delta, \\
& x_{3}:=x^{-}\left(\mathrm{A}^{+}\right) \cap \Delta^{-1} v_{\mathrm{N}}^{\perp} .
\end{aligned}
$$

Denote $\mathrm{A}_{\mathrm{N}}:=\mathrm{A}_{\mathrm{K}_{\mathrm{N}}}$. According to theorem 2.1 we have $x_{1}=x^{+}\left(\mathrm{A}_{\mathrm{N}}\right), x_{2}=x^{0}\left(\mathrm{~A}_{\mathrm{N}}\right)$ and $x_{3}=x^{-}(A)$. Hence $\mathbb{R}^{n}=x_{1} \oplus x_{2} \oplus x_{3}$. With respect to this decomposition we have

$$
A^{-}=\left(\begin{array}{ccc}
A_{11} & 0 & 0 \\
0 & A_{22} & A_{23} \\
0 & 0 & A_{33}
\end{array}\right)
$$

for given matrices $A_{i j}$ This follows from the fact that both $x_{1}$ and $x_{2}$ are $A^{-}$-invariant. Note that $\sigma\left(A_{33}\right) \subseteq \mathbb{C}^{+}$. According to theorem $2.1, x_{2} \oplus x_{3}=$ $\Delta^{-1} x_{1}^{\perp}$. Since also $x_{2}=$ ker $\Delta$, we have

$$
\Delta=\left(\begin{array}{ccc}
\Delta_{11} & 0 & 0 \\
0 & 0 & 0 \\
0 & 0 & \Delta_{33}
\end{array}\right)
$$

with $\Delta_{11}>0$ and $\Delta_{33}>0$. Finally, we partition

$$
B=\left(\begin{array}{l}
B_{1} \\
B_{2} \\
B_{3}
\end{array}\right) \text {. }
$$

Proof of theorem 5.2 From the assumption ker $\Delta \subseteq$ ker $\mathrm{N} \cap$ ker $\mathrm{K}^{-}$it follows that $x_{1} \oplus x_{2} \subseteq \operatorname{ker} \mathrm{N} \cap$ ker $\mathrm{k}^{-}$. Hence $\mathrm{N}$ and $\mathrm{K}^{-}$have the form

$$
\mathrm{N}=\left(\begin{array}{ccc}
0 & 0 & 0 \\
0 & 0 & 0 \\
0 & 0 & \mathrm{~N}_{33}
\end{array}\right), \quad \mathrm{K}^{-}=\left(\begin{array}{lll}
0 & 0 & 0 \\
0 & 0 & 0 \\
0 & 0 & \mathrm{~K}_{33}^{-}
\end{array}\right),
$$

with $\mathrm{N}_{33}-\mathrm{K}_{33}^{-} \geq 0$. Since $\mathrm{K}^{+}=\mathrm{K}^{-}+\Delta$, we have 


$$
\mathrm{K}_{\mathrm{N}}^{+}=\left(\begin{array}{ccc}
0 & 0 & 0 \\
0 & 0 & 0 \\
0 & 0 & \mathrm{~K}_{33}^{-}+\Delta_{33}
\end{array}\right) .
$$

(Recall that $\mathrm{K}_{\mathrm{N}}^{+}=\mathrm{K}^{-} \mathrm{P}+\mathrm{K}^{+}(I-\mathrm{P})$, where $\mathrm{P}$ is the projector onto $x_{1}=V_{\mathrm{N}}$ along $\left.\Delta^{-1} v_{N}^{+}=x_{2} \oplus x_{3}\right)$. By combining the above we see that

$$
K_{N}^{+}-K^{-}=\left(\begin{array}{lll}
0 & 0 & 0 \\
0 & 0 & 0 \\
0 & 0 & \Delta_{33}
\end{array}\right) .
$$

Let $D(t)$ be the solution of the Riccati differential equation (4.2). By lemma 6.4 and the fact that $x_{2}=$ ker $\Delta$ is $A^{-}$-invariant, we have

$$
D(t)=\left(\begin{array}{ccc}
0 & 0 & 0 \\
0 & 0 & 0 \\
0 & 0 & D_{33}(t)
\end{array}\right)
$$

for some $D_{33}(t)$. By writing out $(4.2)$ in the decomposition employed, we see that $D_{33}(t)$ is the unique solution of

$$
\begin{aligned}
& D_{33}(t)=A_{33}^{\top} D_{33}(t)+D_{33}(t) A_{33}-D_{33}(t) B_{3} R^{-1} B_{3}^{\top} D_{33}(t) \\
& D_{33}(0)=N_{33}-K_{33}^{+} .
\end{aligned}
$$

also, $\mathrm{K}_{\mathrm{N}}^{+}-\mathrm{K}^{-}$is a solution of the algebraic Riccati equation (6.3) (see lemma (6.3). This implies that $\Delta_{33}$ is a solution to

$$
A_{33}^{\top} D_{33}+D_{33} A_{33}-D_{33} B_{3} R^{-1} B_{3}^{\top} D_{33}=0
$$

In fact, since $\Delta$ is the largest solution of $(6.3), \Delta_{33}$ is the largest solution of $(6.7)$.

$\left({ }^{\prime} \Rightarrow\right.$ ') Assume that $\mathrm{v}_{\mathrm{T}, \mathrm{N}}^{+}\left(\mathrm{x}_{\mathrm{O}}\right) \rightarrow \mathrm{v}_{\mathrm{N}}^{+}\left(\mathrm{x}_{0}\right)$ for all $\mathrm{x}_{\mathrm{O}}$ or, equivalently, $\mathrm{K}_{\mathrm{N}}(t) \rightarrow \mathrm{K}_{\mathrm{N}}^{+}$ $(t \rightarrow \infty)$. Here, $K_{N}(t)$ is the unique solution of $(4.1)$. Then we have $K_{N}(t)-K^{-} \rightarrow$ $K_{N}^{+}-K^{-}(t \rightarrow \infty)$. Now, the point is that $K_{N}(t)-K^{-}=D(t)$. Hence we find that $D_{33}(t) \rightarrow \Delta_{33}(t \rightarrow \infty)$. Since $\Delta_{33}>0$ it follows from lemma 6.2 that $N_{33}-K_{33}^{-}>0$. We now prove the $A^{-}$-invariance of ker $N \cap$ ker $K^{-}$. Let $x \in \operatorname{ker} N \cap$ ker $K^{-}, x=$ $\left(\mathrm{x}_{1}, \mathrm{x}_{2}, \mathrm{x}_{3}\right)$. Then $\left(\mathrm{N}-\mathrm{K}^{-}\right) \mathrm{x}=0$ whence $\left(\mathrm{N}_{33}-\mathrm{K}_{33}^{-}\right) \mathrm{x}_{3}=0$ so $\mathrm{x}_{3}=0$. Thus $\mathrm{A}^{-} \mathrm{x}=$ $\left(A_{11} x_{1}, A_{22} x_{2}, 0\right)$. Since $x_{1} \oplus x_{2} \subseteq$ ker $\mathrm{N} \cap$ ker $K^{-}$, the claim follows.

$(' \in \prime)$ Assume ker $\mathrm{N} \cap$ ker $\mathrm{K}^{-}$invariant under $\mathrm{A}^{-}$. We then claim that $x_{1} \oplus x_{2}=$ ker $\mathrm{N} \cap$ ker $\mathrm{K}^{-}$. Indeed, 


$$
\begin{aligned}
x_{1} \oplus x_{2} & =\left\{<\operatorname{ker} \mathrm{N} \cap \operatorname{ker} \mathrm{K}^{-} \mid \mathrm{A}^{-}>\cap X^{+}\left(\mathrm{A}^{-}\right)\right\} \oplus \operatorname{ker} \Delta \\
& =\left\{\left(\operatorname{ker} \mathrm{N} \cap \operatorname{ker} \mathrm{K}^{-}\right) \cap X^{+}\left(\mathrm{A}^{-}\right)\right\} \oplus \operatorname{ker} \Delta \\
& =\left(\operatorname{ker} \mathrm{N} \cap \operatorname{ker} \mathrm{K}^{-}\right) \cap\left(\operatorname{ker} \Delta \oplus X^{+}\left(\mathrm{A}^{-}\right)\right),
\end{aligned}
$$

where the last equality again uses the assumption ker $\Delta \subseteq$ ker $K^{-} \cap$ ker $N$. Now, ker $\Delta \oplus X^{+}\left(A^{-}\right)=X^{0}\left(A^{-}\right) \oplus X^{+}\left(A^{-}\right)=\mathbb{R}^{n}$. This proves the claim. It follows from this that $\mathbb{N}_{33}-K_{33}>0$. Hence, since $\sigma\left(A_{33}\right) \subseteq \mathbb{C}^{+}$, the solution $D_{33}(t)$ of $(6.6)$ converges to $\Delta_{33}$, the largest solution of $(6.7)$ (see lemma 6.1). In turn this implies that $\mathrm{D}_{33}(t) \rightarrow \mathrm{K}_{\mathrm{N}}^{+}-\mathrm{K}^{-}$or, equivalently, that $\mathrm{K}_{\mathrm{N}}^{+}(t) \rightarrow \mathrm{K}_{\mathrm{N}}^{+}$. Thus $\mathrm{V}_{\mathrm{T}, \mathrm{N}^{+}}^{+}\left(\mathrm{x}_{0}\right)$ $\rightarrow V_{N}^{+}\left(x_{0}\right)(T \rightarrow \infty)$ for all $x_{0}$. This completes our proof of theorem 5.2.

\section{REFERENCES}

[1] R.w. Brockett, Finite Dimensional Linear Systems, New York, Wiley, 1970.

[2] F.M. Callier \& J.L. Willems, 'Criterion for the convergence of the solution of the Riccati differential equation' IEEE Trans. Aut. Control, AC-26, p. 1232-1242, 1981.

[3] W.A. Coppel, 'Matrix quadratic equation', Bull. Austral. Math Soc., Vol. 10, p. 377-401, 1974.

[4] O. Hijab, Stabilization of Control systems, New York, Springer Verlag, 1987.

[5] R.E. Kalman, 'Contributions to the theory of optimal control', Bol. Soc. Mat. Mex. Vol.5, p. 102-199, 1960.

[6] H. Kwakernaak \& R. Sivan, Linear Optimal Control Systems, Wiley, New York, 1972.

[7] M Shayman, 'Geometry of the algebraic Riccati equation - part 1', SIAM Journal of. Contr. \& Opt., vol. 21, no 3, p. 375-393, 1983.

[8] J.M. Soethoudt \& H.L. Trentelman, 'The regular indefinite linear quadratic problem with linear endpoint constraints', Memorandum Eindhoven University of Technology, 1988, to appear in Systems \& Contr. Letters.

[9] H.L. Trentelman 'The regular free-endpoint linear quadratic problem with indefinite cost', Memorandum Eindhoven University of Technology, 1987 , to appear in SIAM Journal of Cont. \& Opt..

[10] J.C. Willems, 'Least squares stationary optimal control and the algebraic Riccati equation', IEEE Trans. Aut. Control, AC 16, no.6, p. 621-634, 1971.

[11] J.L. Willems \& F.M. Callier, 'Large finite horizon and infinite horizon LQ-optimal control problems', Optimal Contr. Appl. \& Meth., Vol. 4, p $31-45,1983$. 


\section{EINDHOVEN UNIVERSITY OF TECHNOLOGY}

Department of Mathematics and Computing Science

PROBABILITY THEORY, STATISTICS, OPERATIONS RESEARCH AND SYSTEMS THEORY

P.O. Box 513

5600 MB Eindhoven - The Netherlands

Secretariate: Dommelbuilding 0.02

Telephone: $\quad 040-473130$

List of COSOR-memoranda - 1988

\begin{tabular}{llll} 
Number & Month & Author & Title \\
\hline M 88-01 & January & F.W. Steutel, & Haight's distribution and busy periods. \\
& & B.G. Hansen &
\end{tabular}

M 88-02 January J. ten Vregelaar On estimating the parameters of a dynamics model from noisy input and output measurement.

M 88-03 January B.G. Hansen, The generalized logarithmic series distribution.

E. Willekens

M 88-04 January J. van Geldrop, A general equilibrium model of international trade with C. Withagen exhaustible natural resourse commodities.

M 88-05 February A.H.W. Geerts A note on "Families of linear-quadratic problems": continuity properties.

M 88-06 February Siquan, Zhu

A continuity property of a parametric projection and an iterative process for solving linear variational inequalities.

M 88-07 February J. Beirlant, Rapid variation with remainder and rates of convergence.

Willekens

M 88-08 April Jan v. Doremalen, A recursive aggregation-disaggregation method to approxiJ. Wessels mate large-scale closed queuing networks with multiple job types. 
Number Month Author Title

$\begin{array}{ll}\text { M 88-09 April } & \text { J. Hoogendoor, } \\ \text { R.C. Marcelis, } & \text { The Vax/VMS Analysis and measurement packet (VAMP): } \\ \text { A.P. de Grient Dreux, } & \\ \text { J. v.d. Wal, } & \text { R.J. Wijbrands }\end{array}$
M 88-10 April E. Omey,
E. Willekens
Abelian and Tauberian theorems for the Laplace transform of functions in several variables.

$\begin{array}{ll}\text { M 88-11 April } & \text { E. Willekens, } \\ & \text { S.I. Resnick }\end{array}$

E.E.M. v. Berkum

Exact paired comparison designs for quadratic models.

M 88-13 May

J. ten Vregelaar

Parameter estimation from noisy observations of inputs and outputs.

M 88-14 May

L. Frijters,

Lot-sizing and flow production in an MRP-environment.

T. de Kok,

J. Wessels

M 88-15 June

J.M. Soethoudt,

H.L. Trentelman

The regular indefinite linear quadratic problem with linear endpoint constraints.

M 88-16 July

J.C. Engwerda

Stabilizability and detectability of discrete-time time-varying systems.

M 88-17 August $\quad$ A.H.W. Geerts

Continuity properties of one-parameter families of linearquadratic problems without stability.

M 88-18 September W.E.J.M. Bens

Design and implementation of a push-pull algorithm for manpower planning.

M 88-19 September A.J.M. Driessens

Ontwikkeling van een informatie systeem voor het werken met Markov-modellen.

M 88-20 September W.Z. Venema

Automatic generation of standard operations on data structures. 


\begin{tabular}{|c|c|c|c|}
\hline Number & Month & Author & Title \\
\hline \multirow{2}{*}{ M 88-21 } & October & A. Dekkers & Global optimization and simulated annealing. \\
\hline & & E. Aarts & \\
\hline M 88-22 & October & J. Hoogendoorn & Towards a DSS for performance evaluation of VAX/N \\
\hline M 88-23 & October & R. de Veth & $\begin{array}{l}\text { PET, a performance evaluation tool for flexible model } \\
\text { analysis of computer systems. }\end{array}$ \\
\hline M 88-24 & October & J. Thiemann & Stopping a peat-moor fire. \\
\hline M 88-25 & October & $\begin{array}{l}\text { H.L. Trentelman } \\
\text { J.M. Soethoudt }\end{array}$ & $\begin{array}{l}\text { Convergence properties of indefinite linear quadratic } \\
\text { problems with receding horizon. }\end{array}$ \\
\hline
\end{tabular}

